\title{
Congenital prosopagnosia: multistage anatomical and functional deficits in face processing circuitry
}

\author{
V. Dinkelacker $\cdot$ M. Grüter $\cdot$ P. Klaver $\cdot$ \\ T. Grüter $\cdot$ K. Specht $\cdot$ S. Weis $\cdot$ I. Kennerknecht $\cdot$ \\ C. E. Elger $\cdot$ G. Fernandez
}

Received: 6 May 2010/Revised: 30 October 2010/Accepted: 3 November 2010/Published online: 1 December 2010

(C) The Author(s) 2010. This article is published with open access at Springerlink.com

\begin{abstract}
Face recognition is a primary social skill which depends on a distributed neural network. A pronounced face recognition deficit in the absence of any lesion is seen in congenital prosopagnosia. This study investigating 24 congenital prosopagnosic subjects and 25 control subjects aims at elucidating its neural basis with fMRI and voxelbased morphometry. We found a comprehensive behavioral pattern, an impairment in visual recognition for faces and buildings that spared long-term memory for faces with negative valence. Anatomical analysis revealed diminished gray matter density in the bilateral lingual gyrus, the right middle temporal gyrus, and the dorsolateral prefrontal cortex. In most of these areas, gray matter density correlated with memory success. Decreased functional activation was found in the left fusiform gyrus, a crucial area for
\end{abstract}

Electronic supplementary material The online version of this article (doi:10.1007/s00415-010-5828-5) contains supplementary material, which is available to authorized users.

V. Dinkelacker · P. Klaver - C. E. Elger - G. Fernandez

Epileptology, University of Bonn, Bonn, Germany

V. Dinkelacker $(\bowtie)$

Department of Neurophysiology, Hôpital Pitié-Salpêtrière,

47 - 83 rue de l'Hôpital, 75651 Paris, France

e-mail: vdinkel@gmx.de

M. Grüter · I. Kennerknecht

Institute for Human Genetics, University of Muenster,

Muenster, Germany

M. Grüter

Nottulner Landweg, Muenster, Germany

P. Klaver

MR Center, Children's University Hospital and Institute

of Psychology, University of Zurich, Zurich, Switzerland face processing, and in the dorsolateral prefrontal cortex, whereas activation of the medial prefrontal cortex was enhanced. Hence, our data lend strength to the hypothesis that congenital prosopagnosia is explained by network dysfunction and suggest that anatomic curtailing of visual processing in the lingual gyrus plays a substantial role. The dysfunctional circuitry further encompasses the fusiform gyrus and the dorsolateral prefrontal cortex, which may contribute to their difficulties in long-term memory for complex visual information. Despite their deficits in face identity recognition, processing of emotion related information is preserved and possibly mediated by the medial prefrontal cortex. Congenital prosopagnosia may, therefore, be a blueprint of differential curtailing in networks of visual cognition.

Keywords Congenital prosopagnosia - Fusiform gyrus · Emotion · Functional MRI · Voxel-based morphometry

T. Grüter

Department of General Psychology and Methodology, University of Bamberg, Bamberg, Germany

K. Specht

Biological and Medical Psychology, University of Bergen and Haukeland University Hospital, Bergen, Norway

S. Weis

Department of Psychology,

Durham University, Durham, UK

G. Fernandez

Donders Institute for Brain Cognition and Behaviour, Department of Neurology, Radboud University Nijmegen Medical Centre, Nijmegen, The Netherlands 


\section{Introduction}

Face recognition is a key competence in social cognition. Studies on post-traumatic impairments of face recognition provided substantial knowledge of brain regions involved in this highly specialized cognitive function $[1,2]$. In order to assess the genetic and developmental foundations of face recognition, current research interests extend to cases of prosopagnosia in the absence of any lesion, i.e., congenital or developmental prosopagnosia. The prevalence of this condition is about $2 \%$ of either Caucasian or Hong Kong Chinese population [3-6] and it often runs in families. We will henceforth use the term "congenital prosopagnosia (CP)" as it is assumed to be present from birth [7]. In addition to lesion studies, cases of $\mathrm{CP}$ have the potential to unravel fine tuned changes in face processing anatomy and network interaction $[8,9]$. Exploration of this condition covered behavioral, social, anatomical, and fMRI data (for review see [7, 10]). A major concern is a varying degree of heterogeneity in most of the studies reported. While some specific tests have proven useful [11], no single test is able to provide sufficient discriminatory power for the entire entity of prosopagnosia yet.

In the light of this heterogeneity, one obvious solution is to concentrate on single cases with very precise behavioral testing and scrutinize specific functions, e.g., emotion and object processing [12, 13]. An intermediate option is the testing of small groups of CP subjects, a strategy which has been very fruitful for anatomical and electrophysiological changes [14]. Behrmann et al. [15] have laid a foundation of anatomical evidence when showing a decrease in volume of the anterior fusiform gyrus correlating with face recognition impairment. In an intriguing diffusion tensor imaging (DTI) study, Thomas et al. [8] found an impairment of major ventral occipitotemporal white matter tracts. Although heterogeneous within the $\mathrm{CP}$ group, the apparent changes were clearly correlated with behavioral measures. Finally, Garrido et al. [16] undertook a large group study of anatomical changes in developmental prosopagnosic subjects. They found decreased gray matter volume in right anterior inferior temporal lobe and superior temporal sulcus/middle temporal gyrus bilaterally.

Single case and small group studies, however, pose obvious problems for the detection of fMRI activation changes. In a first fMRI study, Avidan and Behrmann [17] found no significant group difference when comparing four prosopagnosic subjects with six control subjects. In a recent report on six subjects [18], they postulated that CP subjects evinced normal adaptation effects in ventral occipito-temporal cortex, while face familiarity effects in prefrontal cortex were absent. The latter finding was not, however, validated by a direct group comparison.
In the light of the methodological constraints depicted above, we opted for a large group size approach based on diagnostic tools developed previously $[19,20]$. This large sample size, similar to Garrido et al. [16], is likely to level out subtle differences in $\mathrm{CP}$ subjects which account for a significant degree of heterogeneity in small group studies. Furthermore, it enables us to screen for widespread changes in cortical density and functional activation, not limited to an a priori hypothesis of occipito-temporal areas known to be involved in $\mathrm{CP}$.

In the current study, we were specifically interested in the processing of facial stimuli with different emotional valence and complex non-face stimuli (buildings). Akin to the anamnestic complaint of prosopagnosic subjects not recognizing people recently encountered, we placed special emphasis on long-term memory of faces and its modulation by emotional valence as we had an a priori knowledge that the emotional valence of a face is correctly recognized [23]. Building facades served as control condition as it was assumed that their recognition would depend more on complex visual analysis akin to face recognition as opposed to nameable object stimuli. A robust blocked design was used to stimulate face processing circuitry and tested long-term memory and categorization of emotional valence after the scanning session.

It was hypothesized that a large group size study would be able to detect alterations in the temporo-occipital core system of face circuitry. In line with recent studies [18], we further conjectured that the extended network of face processing, notably the prefrontal cortex, would be implicated in the disrupted information processing and impair the long-term memory performance. Likewise, it was anticipated that emotional valence, which differentially activates the core and extended system [21, 22], could represent a preserved modality of face related information processing in prosopagnosic subjects [23]. Thus, this study was intended to probe the impact of emotional facial expression on long-term memory success.

\section{Methods}

\section{Subjects}

A total of 49 subjects were included in the present study: 24 of these were congenital prosopagnosic subjects as defined by the criteria described by Grüter et al. [20]. In short, diagnosis was established via a standardized questionnaire and an in-depth semistructured interview the content of which is summarized in Table 1 . These criteria are employed in recent literature on CP [3, 19, 20, 24, 25] as there is currently no single diagnostic test with sufficient sensitivity and specificity. See Gruter and Gruter [26] for a 
Table 1 Characteristics of congenital prosopagnosic (CP) subjects. List of the most discriminative symptoms for the diagnosis of congenital prosopagnosia in the group of 24 prosopagnosic subjects. Symptoms were assessed based on a written questionnaire and an in-depth interview by an experienced physician (MG)

\begin{tabular}{ll}
\hline Diagnosis of CP prior to the study & $\begin{array}{l}\text { Number of subjects } \\
\text { reporting these } \\
\text { symptoms }\end{array}$ \\
\hline Most discriminative symptoms for the diagnosis of CP & \\
Lasting and irritating subjective uncertainty of face recognition & $24 / 24$ \\
Face recognition deficit especially in crowded places or & $24 / 24$ \\
out-of-context encounters & \\
False negative and false positive face recognition events & $24 / 24$ \\
Face recognition time longer than socially accepted & $24 / 24$ \\
Face learning time longer than socially accepted & $24 / 24$ \\
Onset in childhood & $24 / 24$ \\
Development of adaptive behavior & $24 / 24$ \\
No gaze contact necessary in conversation & $24 / 24$ \\
Use of explicit learning strategies for visual person recognition & $24 / 24$ \\
Impaired visual recognition of objects and scenes & $23 / 24$ \\
Affected first degree relatives & $20 / 24$ \\
Cognitive features usually not impaired in prosopagnosics & \\
Normal recognition of facial emotions or emotions in general & $18 / 24$ \\
Unimpaired recognition of gender from faces & $21 / 24$ \\
Normal judgment of facial attractiveness & $24 / 24$ \\
Normal semantic memory for people & $24 / 24$ \\
\hline
\end{tabular}

complete version of the questionnaire with only minor differences to the one used in our study. As four additional $\mathrm{CP}$ subjects had incomplete behavioral testing (encoding or memory), only their anatomical scans were included in the analysis.

Control subjects $(n=31)$ were chosen among university staff and students and underwent the standardized questionnaire in order to exclude prosopagnosia. Three recruited subjects had a dubious questionnaire. They received the semi-structured interview for internal control of the questionnaire (one being a clear $\mathrm{CP}$ subject) and were excluded from the study. Three additional subjects were excluded for technical reasons. Subjects with ophthalmologic impairments, neurological or psychiatric diseases capable of explaining the observed symptoms were excluded from participation in either group. Thus, 25 control subjects remained in the study. The control group was matched to the $\mathrm{CP}$ group for gender, age, education (in years of formal education) and handedness (Edinburgh handedness inventory, see supplementary data). The mean age was $35.6 \pm 8.5$ (range 23-56 years) for prosopagnosic subjects and $34.4 \pm 10.6(20-54$ years $)$ for control subjects.

Both groups comprised 14 women, 1 left-handed and 2 ambidextrous persons. All participants were Caucasian, native German speakers with normal or corrected-to-normal vision. All gave informed consent before participating in the study, which was performed according to the guidelines of the Institutional Ethics Committee. Control subjects were paid $10 € /$ hour, while prosopagnosic subjects were compensated for their travel expenses.

\section{Experimental stimuli}

We tested six different categories of visual stimuli: separate conditions of faces with neutral, negative and positive facial expressions (72 pictures for each category), building facades, scrambled face ellipses, and a fixation cross. Face stimuli were selected from NimStim Face Stimulus Set (http://www.macbrain.org), Pictures of facial affect [27], International Affective Picture Set [28], Karolinska Directed Emotional Faces [29] and freely available internet sources. All pictures represented adult faces with direct eye contact, formatted to a uniform standard (see Fig. 1), half of them were female. The negative condition comprised equal numbers of faces with angry and fearful expressions. The positive condition showed smiling faces. Building facades were selected from freely available photographs (e.g., http://www.photo.net), formatted to the same standard and divided into private $(n=36)$ and public buildings ( $n=36$; offices, factories, etc.). Of the face stimuli, 72 were grossly coarsened with the mosaic function of Adobe Photoshop and half of them rotated into horizontal orientation (Fig. 1). These stimuli were designed to preserve the outline and luminance of a face in the absence of any facial feature, see e.g., [30, 31], in contrast to "scrambled faces" with preserved featural information (see [32] as an example). A white fixation cross served as the resting condition 

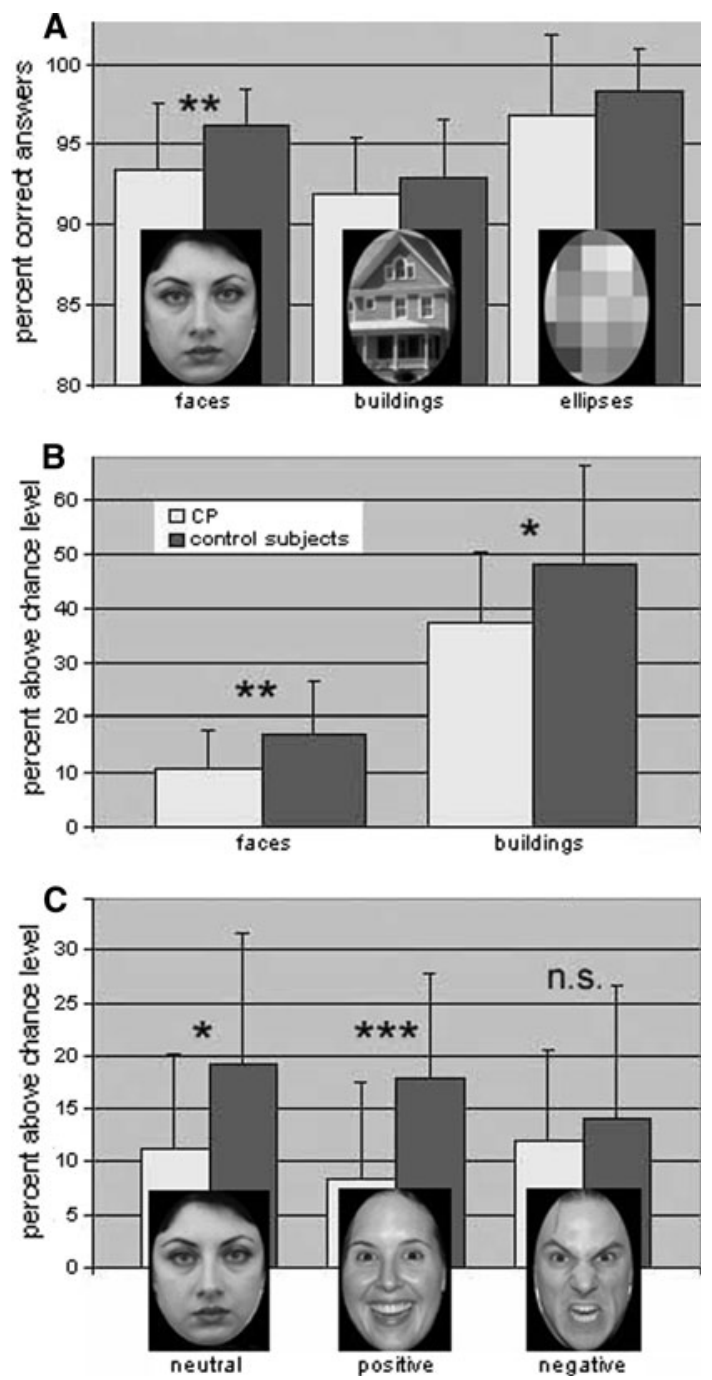

Fig. 1 Behavioral data. Performance of congenital prosopagnosic (CP) subjects was specifically impaired in the gender decision task (a). They showed pronounced impairment for face recognition and for recognition of buildings (b). CP subjects had more difficulties than control subjects when recognizing individuals with neutral and positive facial expressions (c). No difference was found between $\mathrm{CP}$ and control subjects for recognition of individuals with negative facial expressions $(p=0.24)$. Significance level: $* p<0.05, * * p<0.0005$ (see text)

and was continuously presented for one block length (24.12 s).

\section{Experimental procedure}

A blocked design with 12 cycles of each of the following conditions was used: neutral faces, positive faces, negative faces, building facades, scrambled face ellipses, and the resting condition without stimuli. Each cycle consisted of six pictures, with each picture being presented for $3.82 \mathrm{~s}$ (interstimulus interval: $200 \mathrm{~ms}$ ). Stimuli were presented using the Experimental Run-Time System (ERTS, http://www.berisoft.com) and back-projected onto a translucent screen, visible via a mirror mounted on the head coil. Before entering the scanner, subjects had a practice session with 24 additional stimuli. They performed the following tasks via right hand key presses: gender identification for faces, public versus private building for the facades, and horizontal or vertical orientation for scrambled face ellipses. No task was required during resting condition (fixation cross).

After scanning, subjects had a break of $20 \mathrm{~min}$ before continuing with a surprise memory test. On a laptop computer, participants reviewed the pictures they had seen in the scanner (216 faces and 72 buildings), randomly intermixed with 108 new faces and 36 new buildings. During recognition, subjects expressed via self-paced button press whether they had already seen the face with high confidence (category "old"), whether they had not encountered it before (high confidence, category "new") or whether they were uncertain. Uncertain answers were discarded from further analysis. Two response categories determined the accuracy of recognition: the category "hit", a correct "old" response, given with high confidence, and the "false alarm", an erroneous high confidence "old" response for a new item. The probability "hit" is the number of correct "old" responses over all "old" responses required, the probability "false alarm" is the fraction of "old" responses of all "new" responses required. A subject performed at chance level if his or her response "old" was as likely for the old than for the new items (probability hit minus probability false alarm $=0$ ). If this equation yielded a positive result, the subject performed above chance level. After the recognition memory test, subjects were asked to rate the emotional facial expression in a forced choice between happy, neutral, angry, and fearful facial expression. All behavioral data were recorded using ERTS (http://www.erts.de/).

For multiple response possibilities, repeated measures ANOVAs were performed to test for main effects and interactions. In case of significant interactions for the factors group and response category, individual categories were compared by post hoc Student $t$ tests. All statistics beyond imaging data were performed using SPSS. Values are given as mean \pm standard deviation.

fMRI data acquisition

All scans were performed on a $1.5 \mathrm{~T}$ scanner (Symphony, Siemens, Erlangen, Germany) using standard gradients and a circular polarized phase array head coil. Initially, T1weighted 3D-FLASH scans were acquired for anatomical localization and voxel-based morphometry (number of slices: 120; slice thickness: $1.5 \mathrm{~mm}$; interslice gap: none; matrix size: $256 \times 256$; field of view: $230 \mathrm{~mm}$; echo time: 


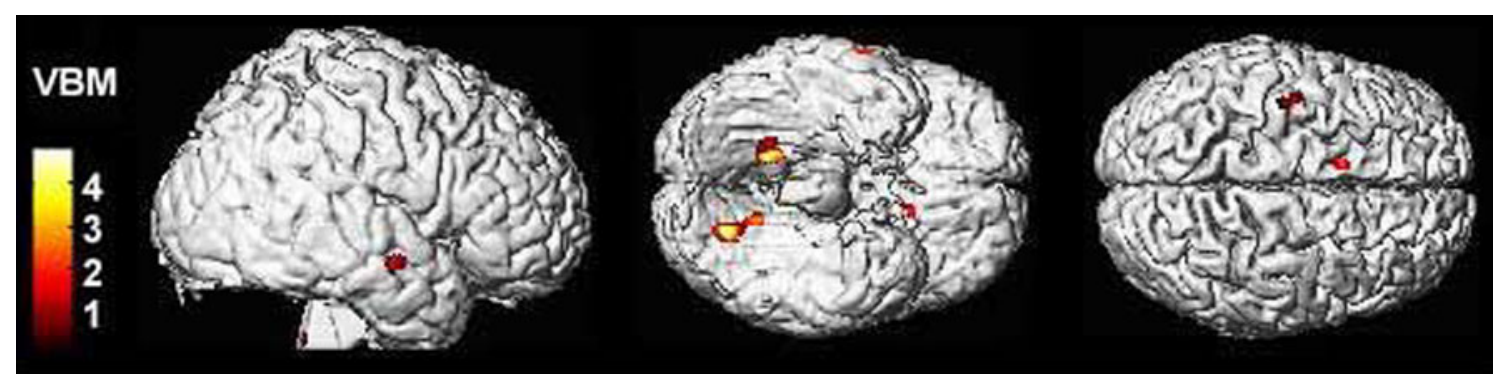

Fig. 2 Widespread areas of decreased cortical density in CP subjects. In a voxel-based morphometry analysis, $\mathrm{CP}$ subjects showed diminished cortical density as compared to control subjects in bilateral

4 ms; repetition time: 11 s). For fMRI, two series of T2*weighted axial EPI scans (including 8 initial dummy scans) were acquired for each subject with the following parameters: number of slices: 30 ; slice thickness: $4 \mathrm{~mm}$; interslice gap: $0.4 \mathrm{~mm}$; matrix size: $64 \times 64$; field of view: $220 \mathrm{~mm}$; echo time: $50 \mathrm{~ms}$; repetition time: $2.52 \mathrm{~s}$. The slice grid for EPI scans was adjusted onto the individual anatomical 3D series in order to ensure complete integration of inferior temporal areas.

\section{Voxel-based morphometry}

Anatomical scans were processed according to previously described protocols [33, 34] using statistical parametric mapping (SPM2, http://www.fil.ion.ucl.ac.uk/spm). This procedure optimizes the normalization for gray matter by the use of a group-specific gray matter template. The preprocessing of the data consists of two major processes: (1) the creation of a template, including new prior-probability maps for gray and white matter, and (2) the normalization and segmentation of the original data for statistical comparisons. As a first step, the anterior commissure was set in each image as the origin of the individual stereotaxic space, followed by a segmentation of the T1-weighted images into gray and white matter probability maps. Only the gray matter maps were normalized to a gray matter template, representing the stereotactical reference space, defined by the Montreal Neurological Institute (MNI). The thereby estimated transformations were applied to the original, non-segmented $\mathrm{T} 1$ dataset, which was then normalized to a voxel size of $2.0 \mathrm{~mm}^{3}$. This normalized $\mathrm{T} 1$ dataset was resegmented and the resulting probability maps for gray matter, white matter, and cerebrospinal fluid were averaged across all subjects and finally smoothed with a Gaussian kernel (full width at half maximum $8 \mathrm{~mm}$ ). These images now served as new group-specific templates and as prior probability maps for gray matter, white matter, and cerebral spinal fluid, which are needed during the segmentation process. These new reference images still represent the MNI space by including individual variations for a better lingual gyrus as well as right middle temporal gyrus and left prefrontal cortex, right cerebellar culmen and left declive $(p<0.001$ unc., see also Table 2)

normalization of high-resolution T1-weighted images. The following procedure is very similar to the one described previously with the exception that now the new reference images are used instead of the standard templates, provided by SPM.

Therefore, we resegmented the original $\mathrm{T} 1$ images, normalized the derived gray matter probability maps to the new gray matter template, applied the thereby estimated transformation to the original $\mathrm{T} 1$ images, resampled it with a voxel-size of $1.0 \mathrm{~mm}^{3}$, segmented the normalized image again, and smoothed the resulting gray matter map with $12 \mathrm{~mm}$. For technical reasons, only 38 subjects were included in this analysis (21 CP subjects (4 with incomplete behavioral testing), 17 control subjects). Their mean image served as a template for Figs. 2, 3, 4 and 5.

The group comparisons were done via two-sample $t$ test. Differences are reported on an uncorrected peak threshold of $p<0.001$ (minimum cluster size 10 voxels). All MNI coordinates were non-linearly transformed to Talairach space via the mni2tal function (http://www.mrc-cbu.cam. ac.uk/Imaging/mnispace.html).

The clusters at the peak threshold level of $p<0.001$ uncorrected (see Fig. 2, right lingual gyrus, left V4v, right middle temporal gyrus, and left dorsolateral prefrontal cortex) were defined as regions of interest via the marsbar function implemented in Matlab (http://www.marsbar. sourceforge.net). For each subject, contrast estimates of enhanced cortical density in control subjects versus prosopagnosic subjects were obtained in the regions of interest in marsbar and exported to SPSS. These values were then tested in SPSS for bivariate correlation (Spearman's) with the marker of long-term memory, i.e., hits minus false alarms in the face or building recognition test.

\section{fMRI data analysis}

MR images were analyzed using SPM2 implemented in MATLAB (Mathworks Inc., Sherborn, MA, USA). All images were realigned to the first image and normalized into standard stereotaxic anatomical MNI space by using 

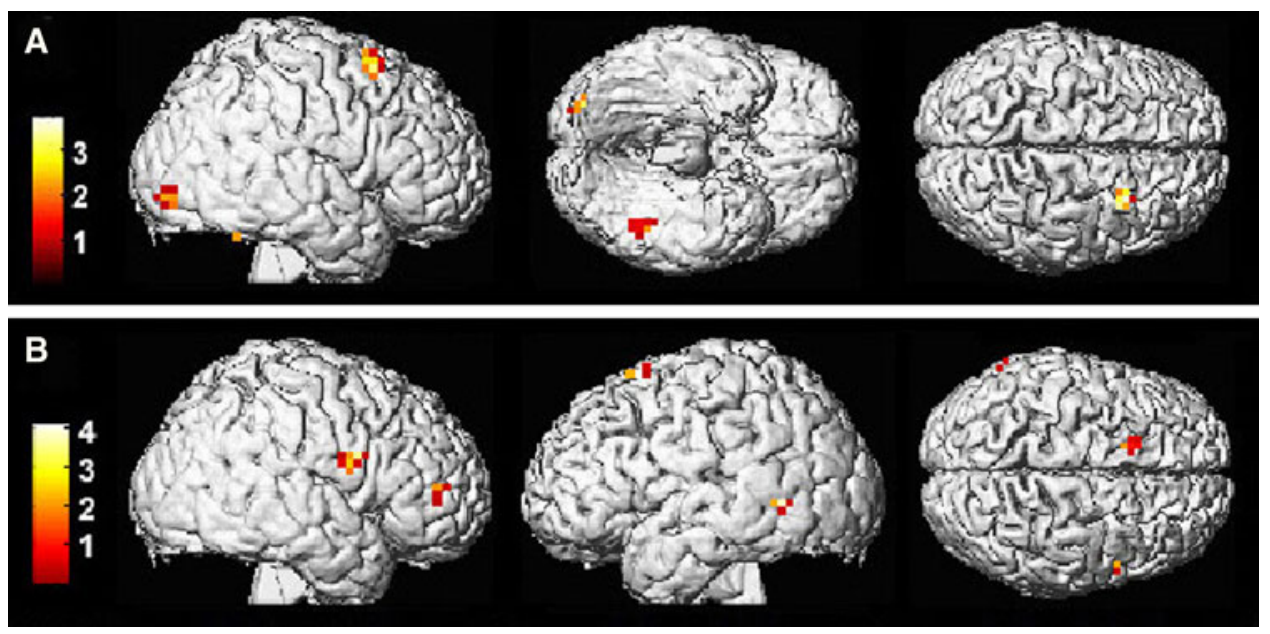

Fig. 3 Deficiencies in classical face and object processing circuitry in $\mathrm{CP}$ subjects. Enhanced activation for control subjects for face (a) and building processing (b). Face processing (faces versus scrambled faces): left fusiform gyrus, right lateral occipital complex (Brodmann area 18/19, inferior occipital gyrus) and right dorsolateral prefrontal cortex (Brodmann area 6). Building processing (buildings versus scrambled faces): left middle temporal gyrus, two areas of ventral prefrontal cortex and a fourth region of the left precentral dorsolateral prefrontal cortex (two sample $t$ tests, peak voxels $p<0.001$ unc., see "Methods" section and Table 3)
Fig. 4 Enhanced activation for $\mathrm{CP}$ subjects in the medial prefrontal cortex. CP subjects showed enhanced BOLD signal in the left medial frontal gyrus and anterior cingulate, Brodmann area 10 (faces versus scrambled faces, two sample $t$ test, peak voxels $p<0.001$ unc.)
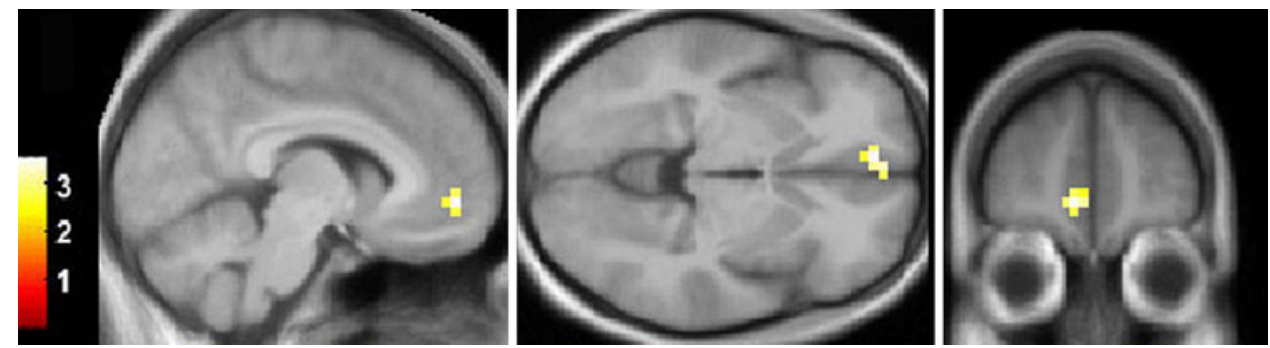
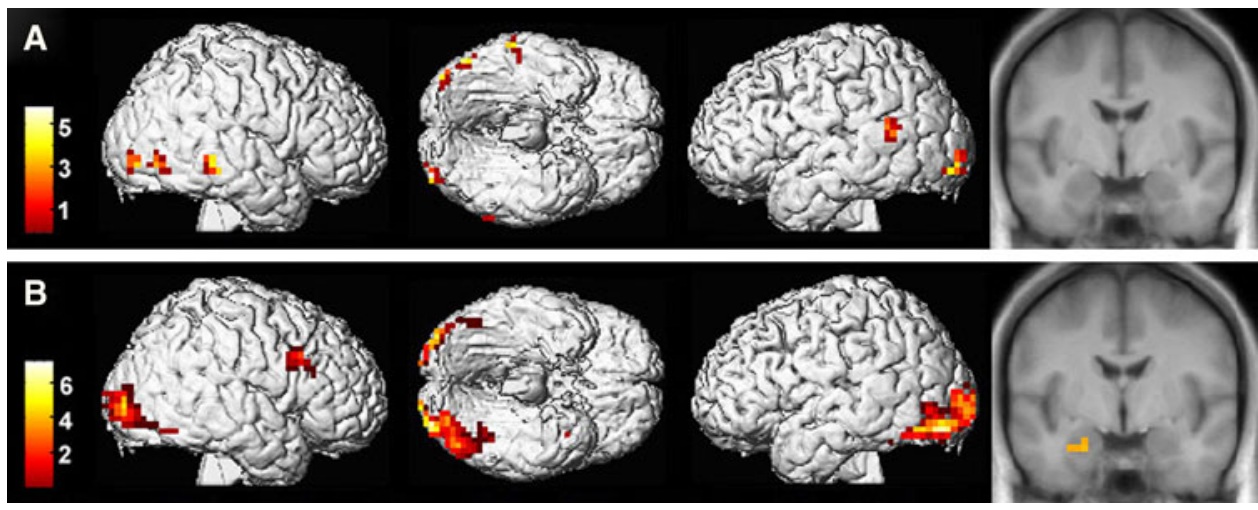

Fig. 5 Different patterns for negative facial expressions in control and $\mathrm{CP}$ subjects. In control subjects (a), the contrast negative versus neutral facial expressions showed pronounced BOLD signal in bilateral lateral occipital complex, left posterior STS, right middle

the transformation matrix calculated from the first EPI scan of each subject and the EPI template. Afterwards, the normalized data with a resliced voxel size of $4 \mathrm{~mm}^{3}$ were smoothed with an 8-mm FWHM isotropic Gaussian kernel. Proportional scaling with high pass filtering was used to eliminate confounding effects of differences in global temporal gyrus; amygdala below threshold ( $p<0.001$ unc.). In CP subjects (b), the same contrast elicited a general enhancement of activation in bilateral temporo-occipital cortex, right ventrolateral prefrontal cortex, and left amygdala

activity within and between subjects. The data were analyzed using a general linear model where the different experimental conditions were modeled as boxcar functions, convolved with the canonical hemodynamic response function (HRF) and its temporal derivative. Subsequently, parameter estimates of the HRF regressor for each of the 
different conditions were calculated from the least mean squares fit of the model to the time series. Parameter estimates for the temporal derivative were not further considered in any contrast. Estimation of the model included correction for non-sphericity induced to the design by the non-independent and non-identically distributed errors of the parameter estimates [35].

One way ANOVAs for the group of prosopagnosic subjects or for the group of control subjects were used when contrasting emotional and neutral facial expressions, at a peak threshold level of $p<0.001$ uncorrected (Fig. 5). When comparing group differences between prosopagnosic subjects and control subjects, a random effects analysis was performed using two sample $t$ tests. Here, the reported significance of the peak threshold is $p<0.005$, cluster size 5 voxels, all including at least two peak activity voxels with a significance threshold of $p<0.001$ uncorrected unless otherwise noted. Activations are shown overlaid onto a canonical brain without cerebellum rendered in three dimensions as well as projected onto coronal slices of the anatomic mean of 38 subjects. The reported voxel coordinates of activation peaks are given in Talairach coordinates in Table 3.

\section{Results}

\section{Behavioral data}

\section{Face and building discrimination tasks}

During fMRI scanning, subjects performed a gender decision task for all faces. For building facades, they assessed whether they saw a private or a public building. For scrambled face ellipses an orientation decision (horizontal or vertical) was required. This orientation task was designed to be feasible for both groups; thus, all performances ranged close to ceiling levels (see Fig. 1a).

Differences in task difficulty still caused a main effect for stimulus category $\left(F_{2,94}=35.63 ; p<0.001\right)$ in a repeated measures ANOVA, which also revealed a significant group effect $\left(F_{1,47}=5.17 ; p=0.028\right)$. The latter is difficult to interpret in the light of ceiling effects and in the absence of interaction with stimulus category $(p>0.05)$. However, it seems to be primarily mediated by a deficit in gender processing: prosopagnosic participants identified $93 \pm 4 \%$ of faces correctly as female/male as compared to $96 \pm 2 \%$ in control subjects (a $t$ test would yield $t=2.91$, degrees of freedom $(d f)=35.9, p<0.005$ but no difference for buildings or ellipses, Fig. 1a).

Analysis of reaction times showed a significant main effect of task $\left(F_{2,46}=217.4 ; p<0.0001\right)$ as well as an interaction of group with stimulus category $\left(F_{2,46}=4.56\right.$; $p=0.016$ ), but no main effect of group. Post hoc $t$ tests revealed that prosopagnosic subjects were faster for the orientation decision of ellipses $(1,086 \pm 150 \mathrm{~ms}$ vs. $1,175 \pm 195 \mathrm{~ms}, t=1.80, d f=44.8, p<0.05)$ and in tendency faster for buildings $(1,598 \pm 232 \mathrm{~ms}$ vs. $1,701 \pm$ $262 \mathrm{~ms}, p=0.08)$, without noticeable differences for the gender decision $(p=0.38)$. Differences in face recognition memory should, therefore, not be biased by the reaction times during implicit encoding.

In summary, correct answers in the orientation task were close to ceiling levels, but detailed analysis suggests a subtle overall performance deficit in CP subjects, possibly driven by difficulties in the gender decision. For the nonface stimuli, CP subjects were faster than control subjects.

\section{Impaired memory for faces and buildings in CP subjects}

Subjects were confronted with a surprise recognition memory test 20 min after scanning. Memory performance was determined by the probability of a correct recognition ("hit") versus the probability of a false recognition (see "Methods"). Here, we found a main effect for stimulus category $\left(F_{1,47}=258, p<0.001\right)$ and a significant group effect $\left(F_{1,47}=7.67, p=0.008\right)$ revealing a memory impairment for both faces and building facades in $\mathrm{CP}$ subjects (Fig. 1b). Yet, there was no significant interaction between stimulus category and group. For faces, prosopagnosic subjects scored $10 \pm 7 \%$ above chance level. Control subjects attained $17 \pm 10 \%$, a difference which was significant in a planned $t$ test $(t=2.73, d f=43.3$, $p<0.005$, Fig. 1b, buildings: $t=2.39, \quad d f=43.4$, $p<0.05)$. In addition, $\mathrm{CP}$ subjects were more likely to answer "uncertain" when asked to recognize faces (41 \pm $2 \%$ of all answers vs. $31 \pm 2 \%, t=2.31, d f=40.8$, $p<0.05$ ), which also held in tendency when recognizing buildings $(28 \pm 2 \%$ vs. $21 \pm 1 \%$ in control subjects, $p=0.06$ ). Note that uncertain answers were not included in the above behavioral analyses. Hence, even when CP subjects felt confident, their recognition memory was significantly worse compared to control subjects.

\section{Normal memory for faces with negative facial expression in CP subjects}

The memory success for happy, neutral, and negative facial expressions were also determined. A repeated measure ANOVA revealed a significant interaction between the factors memory success per emotional valence and group $\left(F_{2,46}=3.37 ; p=0.043\right)$ and a group effect $\left(F_{1,47}=7.48\right.$; $p=0.009)$, but there was no significant effect of stimulus category. Post hoc $t$ tests showed that face recognition was deficient in CP subjects both for neutral and positive facial expressions $(t=2.65, d f=44.0, p<0.01$ and $t=3.52$, 
Table 2 Voxel-based morphometry data. Areas of diminished gray matter density in CP as compared to control subjects with $t$ and $p$ values (unc. $=$ uncorrected) as well as Talairach coordinates $(x, y, z$ in $\mathrm{mm})$

\begin{tabular}{|c|c|c|c|c|c|c|c|}
\hline VBM analysis & Brain region & $\begin{array}{l}\text { Cluster size } \\
\text { in voxels }\end{array}$ & $t$ value & $p$ value (unc.) & $x(\mathrm{~mm})$ & $y(\mathrm{~mm})$ & $z(\mathrm{~mm})$ \\
\hline \multirow{12}{*}{$\begin{array}{l}\text { Diminished gray } \\
\text { matter density in } \mathrm{CP}\end{array}$} & \multirow[t]{3}{*}{ Right lingual gyrus } & \multirow[t]{3}{*}{1,434} & 4.83 & 0.000 & 13 & -50 & -11 \\
\hline & & & 3.93 & 0.000 & 23 & -50 & -14 \\
\hline & & & 3.88 & 0.000 & 16 & -47 & -5 \\
\hline & \multirow[t]{3}{*}{ Left lingual gyrus } & \multirow[t]{3}{*}{1,141} & 4.55 & 0.000 & -21 & -69 & -8 \\
\hline & & & 3.85 & 0.000 & -15 & -54 & -7 \\
\hline & & & 3.44 & 0.001 & -16 & -61 & -10 \\
\hline & Right middle temporal gyrus & 141 & 4.23 & 0.000 & 67 & -6 & -12 \\
\hline & Left precentral & 151 & 4.19 & 0.000 & -43 & -8 & 59 \\
\hline & Left prefrontal & 161 & 4.03 & 0.000 & -12 & 15 & 46 \\
\hline & Left orbitofrontal & 133 & 3.54 & 0.001 & -10 & 16 & -24 \\
\hline & Left cerebellum & 58 & 3.56 & 0.001 & -13 & -44 & -36 \\
\hline & Right cerebellum & 43 & 3.49 & 0.001 & 54 & -51 & -25 \\
\hline
\end{tabular}

$d f=47.0, p<0.0005$, respectively, see Fig. 1c). However, CP subjects performed equally well when it came to recognizing individuals with negative facial expressions $(p=0.24)$. Within the control group, repeated measures ANOVA revealed no significant effect of memory success per emotional category. This was, however, in tendency the case for CP subjects $\left(F_{2,22}=3.34 ; p=0.054\right)$. Prosopagnosic subjects remembered faces with negative (and neutral) valence in tendency better than faces with positive valence (post hoc $t$ tests would be $p<0.05$ significant, Fig. 1c, white bars). For the recognition memory task, no main effect of group or interaction with group was noted.

\section{Normal rating of the emotional valence in CP subjects}

Concomitant to the self-paced face recognition memory test, subjects had to decide for each face whether its expression was neutral, happy, angry or fearful. CP subjects accomplished this task equally well as the control subjects. They attained $94 \pm 4 \%$ correct emotional classification as compared to $92 \pm 10 \%$ in control subjects (repeated measures ANOVA non significant). On a behavioral level, the emotional valence of a face is, thus, correctly categorized and, if negative, contributes to a recognition memory performance comparable to control subjects.

\section{Anatomical differences and their relation to memory success}

Voxel-based morphometry revealed diminished gray matter density in CP subjects in a distributed network. Main clusters span bilateral lingual gyri, extending in the left hemisphere into a posterior area compatible with V4v (Fig. 2; Table 2). Notably, parts of these clusters are projected onto both cerebellar hemispheres (see Table 2). Further areas of decreased gray matter density include the right middle temporal gyrus, the left precentral gyrus (Brodmann area 6), and a left prefrontal region (medial frontal gyrus, adjacent to Brodmann area 6, Fig. 2; Table 2). Note that not all of the subjects were included in this analysis (see "Methods"). There is no difference in overall gray and white matter volume between the two groups.

How do the anatomical differences between CP subjects and control subjects relate to the behavioral outcome? Our most robust measure of impaired face recognition is the longterm memory, i.e. the percentage hits minus false alarms for each subject collapsed over the different emotional valences. In a bivariate correlation analysis (Spearman's) between contrast estimate and memory outcome over all subjects, two areas of decreased cortical density were closely linked to face memory success, namely the left lingual gyrus (Spearman's correlation coefficient $\rho=0.312 ; p<0.05)$ and the left dorsolateral prefrontal cortex (DLPFC, $\rho=0.432$, $p<0.01)$. In the latter area, this correlation was also significant within the prosopagnosia group itself $(p<0.05)$, indicating that the DLPFC is important for their face memory. A further anatomical cluster, the right middle temporal gyrus, was correlated with the recognition performance for buildings over all subjects ( $\rho=0.502 ; p<0.005)$, which held in tendency when calculated only in CP subjects $(p=0.062)$.

\section{fMRI data}

\section{Enhanced activation for control subjects in landmarks of visual circuitry}

The comparison of face processing between $\mathrm{CP}$ subjects and control subjects yielded three distinct areas of decreased activity in CP subjects: the left fusiform gyrus and the right lateral occipital complex in the core face 
Table 3 fMRI data. Activation differences between CP and control subjects with $t$ and $p$ values (unc. = uncorrected) as well as Talairach coordinates $(x, y, z$ in $\mathrm{mm})$ for fMRI data

\begin{tabular}{|c|c|c|c|c|c|c|c|}
\hline fMRI analysis & Brain region & $\begin{array}{l}\text { Cluster size } \\
\text { in voxels }\end{array}$ & $t$ value & $p$ value (unc.) & $x(\mathrm{~mm})$ & $y(\mathrm{~mm})$ & $z(\mathrm{~mm})$ \\
\hline \multirow{3}{*}{$\begin{array}{l}\text { Enhanced activation for } \\
\text { control subjects, faces } \\
\text { versus scrambled faces }\end{array}$} & Right lateral occipital complex & 11 & 3.10 & 0.002 & 24 & -86 & -2 \\
\hline & Left fusiform gyrus & 16 & 3.44 & 0.005 (4 voxels 0.001$)$ & -44 & -52 & -21 \\
\hline & Right dorsolateral prefrontal cortex & 21 & 3.75 & $\begin{array}{l}0.005 \\
(4 \text { voxels } 0.001)\end{array}$ & 28 & 18 & 54 \\
\hline \multirow{2}{*}{$\begin{array}{l}\text { Enhanced activation for } \\
\text { control subjects, negative } \\
\text { faces versus scrambled } \\
\text { faces }\end{array}$} & Left fusiform gyrus & 18 & 3.76 & 0.005 (6 voxels 0.001$)$ & -44 & -48 & -21 \\
\hline & Right dorsolateral prefrontal cortex & 10 & 3.74 & 0.005 (4 voxels 0.001$)$ & 28 & 18 & 54 \\
\hline \multirow{4}{*}{$\begin{array}{l}\text { Enhanced activation for } \\
\text { control subjects, buildings } \\
\text { versus scrambled faces }\end{array}$} & Left precentral & 7 & 4.12 & 0.000 & -16 & 16 & 64 \\
\hline & Left middle temporal gyrus & 7 & 3.60 & 0.000 & -60 & -56 & 0 \\
\hline & Right ventro-lateral pre-frontal & 13 & 3.42 & 0.001 & 48 & 4 & 20 \\
\hline & Right fronto polar & 5 & 3.10 & 0.002 & 36 & 48 & 8 \\
\hline $\begin{array}{l}\text { Enhanced activation for } \mathrm{CP} \text {, } \\
\text { faces versus scrambled } \\
\text { faces }\end{array}$ & Left medial prefrontal & 11 & 3.55 & 0.005 ( 2 voxels 0.001$)$ & -8 & 50 & -6 \\
\hline \multirow{2}{*}{$\begin{array}{l}\text { Enhanced activation } \mathrm{CP} \text {, } \\
\text { negative faces versus } \\
\text { scrambled faces }\end{array}$} & \multirow[t]{2}{*}{ Medial prefrontal } & \multirow[t]{2}{*}{19} & 3.72 & \multirow[t]{2}{*}{0.001 (7 voxels 0.001$)$} & -8 & 50 & -6 \\
\hline & & & 3.39 & & 4 & 54 & -3 \\
\hline
\end{tabular}

processing areas of the ventral occipito-temporal cortex, and the right DLPFC. These areas are illustrated in Fig. 3a (faces versus scrambled faces, Brodmann area 18/19; for all Talairach coordinates see Table 2). The activation in the fusiform gyrus also encompasses more caudal areas like the left cerebellar hemisphere, which is not an uncommon finding in face processing (Table 3, see "Discussion"). The right DLPC represented the largest cluster, its coordinates being very much akin to the contralateral decrease in cortical density (Brodmann area 6, Fig. 3a).

What were the differences for object stimuli? When contrasting buildings with visual control stimuli, our data are again most compatible with a dysfunctional network. CP subjects showed diminished BOLD signals in left middle temporal gyrus, in two areas of ventral prefrontal cortex and a fourth region of the left precentral DLPFC (Fig. 3b). This pattern of functional differences in the processing of buildings mirrors two locations of the anatomical changes in $\mathrm{CP}$ subjects: the middle temporal gyrus, where anatomical changes correlated with memory for buildings and the precentral DLPFC. The latter region emerges in the four axes of our data analysis: the study of cortical density, in the correlation with face and building memory, and here in functional face and building processing.

\section{Enhanced activation for CP subjects in medial prefrontal cortex}

When regarding enhanced activation in participants with $\mathrm{CP}$ (faces versus scrambled faces), only one brain region came into play: the left medial prefrontal cortex (Fig. 4, contrast faces vs. scrambled faces, Brodmann area 10). This area is commonly associated with emotional processing, notably of negative facial expressions [36]. Accordingly, this enhanced activation in CP subjects appeared even more pronounced for negative faces (see Table 2). The contrast negative versus neutral faces did not yield significant group differences. It was suggestive, though, of a substantial overall enhancement of face responsive activity in $\mathrm{CP}$ subjects by negative facial expression (Fig. 5b). The activation in the $\mathrm{CP}$ group included the left amygdala and extensive ventral occipitotemporal cortex in line with an overall enhancement of face sensitive areas by fearful faces [62]. Behavioral and functional data in $\mathrm{CP}$ subjects, therefore, suggest a preserved processing of emotional information. Control subjects showed a weaker effect, most obvious in the occipital cortex, the right middle temporal gyrus, and left posterior STS (Fig. 5a). Note that CP subjects did not show any preferentially engaged area in the processing of buildings.

\section{Discussion}

Our behavioral and imaging data evince that people with $\mathrm{CP}$ are impaired in long-term memory for faces and, to a lesser extent, other complex visual stimuli such as buildings. However, their memory for faces with negative valence and the ability to categorize emotional expressions are preserved. The comparison of cortical density revealed 
structural deficits in medial inferior temporal areas, notably in the lingual gyrus, in right middle temporal cortex, and DLPFC. In most of these areas, gray matter density correlated with memory success. CP subjects showed decreased BOLD signal during face processing in the fusiform gyrus, lateral occipital complex, and right DLPFC. Processing of buildings was accompanied by decreased brain activity in middle temporal gyrus, ventral and precentral DLPFC. Thus, both structural and functional deficits in the DLPFC emerged in different lines of analysis. Prosopagnosic subjects, on the other hand, showed enhanced activation in the medial prefrontal cortex.

Impaired memory for complex neutral stimuli but not for negative faces

The behavioral results revealed diminished long-term memory for faces with neutral and happy expressions, in close keeping with the pivotal complaint of CP subjects in everyday life. Although face memory deterioration is the most prominent behavioral impairment, the memory for complex objects such as buildings is not spared [37]. Here we could show a clear performance deficit in CP subjects both for faces and building facades. Data on CP subjects regarding deficits in object recognition is not homogeneous [38-40]. However, in a family with ten prosopagnosic subjects, recognition impairment was found to extend to categories such as cars and guns [41].

To our knowledge, this is the first study to probe longterm memory for emotional faces. Interestingly, recognition of faces with negative expressions is preserved in $\mathrm{CP}$ subjects. Emotional categorization [42] and fear related memory is obviously intact in CP subjects. This finding will be discussed in conjunction with the functional data below.

In the orientation task, people with $\mathrm{CP}$ have a discreet overall disadvantage in the face and object tasks, which was most obvious for the gender decision. This is in line with earlier study results [38]. Note, however, that reaction times were faster for CP subjects in object-related tasks. Hence, their deficits are best explained by a circumscribed impairment in the processing and recognition memory for complex visual stimuli [41].

Voxel-based morphometry: anatomical changes in a distributed visual network

\section{Lingual gyrus}

Voxel-based morphometry revealed widespread areas of diminished gray matter density. Deficits were most prominent in bilateral lingual gyrus and were correlated with face memory success, suggesting that these cortical zones are actively engaged in face processing tasks. In terms of Talairach coordinates (right: $13,-50,-11$; left: $-15,-54$, $-7)$, they are well compatible with the building-specific region in the right lingual gyrus $(20.6 \pm 5.0 ; 53.8 \pm 6.7$; $-9.2 \pm 3.9$ [43]) and with the parahippocampal place area. Note that the topography of face and building sensitive areas is not only a product of object class but also markedly biased by peripheral visual processing. Levy et al. [44, 45] concluded that the processing of buildings relies more on peripheral vision. With controlled stimuli, they demonstrated that central perception of both building and face information activates lateral portions of inferior temporal cortex (often implicated in face processing), whereas peripheral vision engages the more medial areas, topographically close to the diminished cortical clusters of lingual gyrus in CP subjects. So we may speculate that decreased cortical density in this region is related to general perceptual differences in $\mathrm{CP}$ subjects. The gray matter deficits both in lingual and in middle temporal gyrus are well in line with a recent large study on gray matter volume in developmental prosopagnosic subjects, where the right lingual gyrus was deficient at the uncorrected level, yet it was not included in small volume correction masks (Garrido et al. [16], supplementary data).

\section{Middle temporal gyrus}

A further cluster of decreased cortical density lay in right middle temporal gyrus and correlated with long-term memory for buildings. This area, which also showed diminished BOLD signal in building processing in $\mathrm{CP}$ subjects (Fig. 3b), has multimodal functional tasks [46] and was implicated in naming famous faces and buildings [47]. Possibly, its role in configural analysis, as demonstrated for faces by Lobmaier et al. [32], may add to its importance in building and face identification. This finding fits nicely to the study by Garrido et al. [16]: the gray matter volume of the right superior temporal sulcus/middle temporal gyrus emerged as the most significant difference between developmental prosopagnosic subjects and controls. They found it to be associated with a behavioral component related to face identity, in keeping with its role as a face sensitive area.

\section{Dorsolateral prefrontal cortex}

In the current study, bilateral involvement of precentral DLPFC emerged in anatomical, behavioral, and functional lines of analysis. This finding supports the hypothesis that the disruption of information propagation to the extended system of face processing has a crucial pathophysiological role in $\mathrm{CP}$ [8, 18]. Thomas et al. [8] found alterations in major inferior temporal fiber connections, namely the 
inferior fronto-occipital fasciculus in a study with six $\mathrm{CP}$ subjects. Interestingly, this fasciculus connects most of the deficient areas we found (e.g., the lingual, fusiform, and inferior temporal gyri) with the inferolateral frontal cortex [48]. It is a part of the fronto-occipital fasciculus which projects to areas 6, 8 and 9 in DLPFC [49]. We may, therefore, speculate that prosopagnosic subjects suffer from an impairment of face processing circuitry that affects the integrity of both white matter tracts and dependant cortical areas. This disruption also compromises the activation of major network nodes.

Differential fMRI activation reveals a putative alternative route

\section{Inferior temporal cortex}

Face and building processing in $\mathrm{CP}$ subjects was accompanied by decreased functional activity in visual circuitry. During the processing of faces of all valences, the left fusiform gyrus was less active in $\mathrm{CP}$ subjects. This activation is topographically compatible with the fusiform face area (FFA), which was not separately determined in our study. The region of decreased activity comprises more caudal areas extending into the cerebellum, which is not an uncommon finding in previously described FFA activations (Table 2, see [50, 51] for comparison). Implication of this region in $\mathrm{CP}$ is, hence, in agreement with the pivotal role of the FFA in face-related tasks [21, 52, 53]. Diminished FFA activation has been reported in small samples of $\mathrm{CP}$ subjects [54, 55], but its significance is a matter of debate. Avidan and Behrmann [18] reported a weaker signal for right and most notably left FFA in CP subjects, but as its modulation by repetition of faces was normal, they considered the disruption of information propagation to the prefrontal extended system as more significant.

\section{Dorsolateral prefrontal cortex}

The prefrontal cortex is differentially activated in $\mathrm{CP}$ subjects: BOLD signal is diminished in DLPFC and increased in medial prefrontal cortex. The DLPFC and the adjacent premotor cortex have a role in facial working memory [56-58] and contribute via complex mechanisms to long-term memory [59], while bolstering rather a feeling of familiarity than conscious recollection [60]. The DLPFC has been associated with relational working memory encoding of visual items [61], a task which draws heavily on mental imagery know to be deficient in CP subjects [37, 62]. As the cortical density in a left DLPFC area correlated with recognition memory performance and DLPFC activation was decreased in $\mathrm{CP}$ subjects, we suggest that DLPFC impairment contributes to the long-term memory deficits in $\mathrm{CP}$ subjects. The altered gaze behavior towards faces in CP subjects [67] may also indicate an executive dysfunction of the visual system mediated by the DLPFC and adjacent premotor cortex $[63,64]$. However, the exact nature of the DLPFC malfunction in $\mathrm{CP}$ remains unclear. It may be the primary cause of the condition or a consequence of deficits in other parts of the visual system.

\section{Medial prefrontal cortex}

The medial prefrontal cortex is significantly more engaged in $\mathrm{CP}$, both during general face processing and when processing negative facial expressions. This discrepancy to the study by Avidan and Behrmann [18], who showed anterior cingulate activation in control subjects but not in CP subjects, may be due to our larger group size, which allows for direct group comparison. In addition, we used unfamiliar and emotional faces, which elicit more amygdala and orbitofrontal activation [21, 65]. Both areas have been shown to be strongly interconnected $[65,66]$. Implication of the extended system of face processing [18] in CP subjects would, hence, depend on the emotional valence of the visual input. As several prefrontal regions, namely the anterior cingulate and rostral prefrontal cortex, are important for successful recognition memory for emotional faces [67], we may speculate that prosopagnosic subjects rely on extraction of emotional cues via the medial prefrontal cortex, which may help to preserve their long-term memory for emotional faces. Yet, our study cannot tap further into this question due to methodological constraints. The fact that we allowed an initial response option "uncertain" in the memory task resulted in a considerable limitation of the analysis. The number of discarded trials varied between subjects (see supplementary data), thus, hampering the detailed analysis of memory performance and the subsequent memory effect in fMRI. Still we feel inclined to hypothesize that a concurrent network of emotional processing via the medial prefrontal cortex is intact in $\mathrm{CP}$ subjects.

\section{Conclusion}

The symptoms of congenital prosopagnosia are best explained by a dysfunctional network of face processing which likely comprises both the core and the extended system of visual circuitry. In the core system, anatomical changes are most apparent in the lingual gyrus and in the middle temporal gyrus, two areas commonly involved in face and building processing. Functional deficits further extend to major landmarks of face circuitry, such as fusiform gyrus, lateral occipital complex, and middle temporal gyrus. Furthermore, our data unravels the differential implication of the extended system in prefrontal cortex. 
Thus, the long-term memory impairment for neutral material may be explained by insufficient involvement of DLPFC. Conversely, prosopagnosic subjects are able to compensate for these deficits in emotion related task, possibly via an alternative route to the medial prefrontal cortex. This alternative route warrants further scrutiny.

Acknowledgments This work was supported by a Gerok grant from the Deutsche Forschungsgemeinschaft for Vera Dinkelacker.

Open Access This article is distributed under the terms of the Creative Commons Attribution Noncommercial License which permits any noncommercial use, distribution, and reproduction in any medium, provided the original author(s) and source are credited.

\section{References}

1. Bouvier SE, Engel SA (2006) Behavioral deficits and cortical damage loci in cerebral achromatopsia. Cereb Cortex 16:183-191

2. Damasio AR, Damasio H, Van Hoesen GW (1982) Prosopagnosia: anatomic basis and behavioral mechanisms. Neurology 32:331-341

3. Kennerknecht I, Grueter T, Welling B, Wentzek S, Horst J et al (2006) First report of prevalence of non-syndromic hereditary prosopagnosia (HPA). Am J Med Genet A 140:1617-1622

4. Kennerknecht I, Ho NY, Wong VC (2008) Prevalence of hereditary prosopagnosia (HPA) in Hong Kong Chinese population. Am J Med Genet A 146A:2863-2870

5. Kennerknecht I, Pluempe N, Welling B (2008) Congenital prosopagnosia-a common hereditary cognitive dysfunction in humans. Front Biosci 13:3150-3158

6. Kennerknecht I, Plumpe N, Edwards S, Raman R (2007) Hereditary prosopagnosia (HPA): the first report outside the Caucasian population. J Hum Genet 52:230-236

7. Behrmann M, Avidan G (2005) Congenital prosopagnosia: faceblind from birth. Trends Cogn Sci 9:180-187

8. Thomas C, Avidan G, Humphreys K, Jung KJ, Gao F et al (2009) Reduced structural connectivity in ventral visual cortex in congenital prosopagnosia. Nat Neurosci 12:29-31

9. Grüter T, Carbon CC (2010) Neuroscience. Escaping attention. Science 328(5977):435-436

10. Dobel C, Bolte J, Aicher M, Schweinberger SR (2007) Prosopagnosia without apparent cause: overview and diagnosis of six cases. Cortex 43:718-733

11. Duchaine B, Nakayama K (2006) The Cambridge Face Memory Test: results for neurologically intact individuals and an investigation of its validity using inverted face stimuli and prosopagnosic participants. Neuropsychologia 44:576-585

12. Duchaine BC, Parker H, Nakayama K (2003) Normal recognition of emotion in a prosopagnosic. Perception 32:827-838

13. Duchaine BC, Dingle K, Butterworth E, Nakayama K (2004) Normal greeble learning in a severe case of developmental prosopagnosia. Neuron 43:469-473

14. Dobel C, Putsche C, Zwitserlood P, Junghofer M (2008) Early left-hemispheric dysfunction of face processing in congenital prosopagnosia: an MEG study. PLoS ONE 3:e2326

15. Behrmann M, Avidan G, Gao F, Black S (2007) Structural imaging reveals anatomical alterations in inferotemporal cortex in congenital prosopagnosia. Cereb Cortex 17(10):2354-2363
16. Garrido L, Furl N, Draganski B, Weiskopf N, Stevens J et al (2009) Voxel-based morphometry reveals reduced grey matter volume in the temporal cortex of developmental prosopagnosics. Brain 132:3443-3455

17. Avidan G, Hasson U, Malach R, Behrmann M (2005) Detailed exploration of face-related processing in congenital prosopagnosia: 2. Functional neuroimaging findings. J Cogn Neurosci 17:1150-1167

18. Avidan G, Behrmann M (2009) Functional MRI reveals compromised neural integrity of the face processing network in congenital prosopagnosia. Curr Biol 19:1146-1150

19. Grueter M, Grueter T, Bell V, Horst J, Laskowski W et al (2007) Hereditary prosopagnosia: the first case series. Cortex 43:734-749

20. Grüter T, Grüter M, Carbon CC (2008) Neural and genetic foundations of face recognition and prosopagnosia. J Neuropsychol 2(Pt 1):79-97

21. Haxby JV, Hoffman EA, Gobbini MI (2000) The distributed human neural system for face perception. Trends Cogn Sci 4:223-233

22. Gobbini MI, Haxby JV (2007) Neural systems for recognition of familiar faces. Neuropsychologia 45:32-41

23. Humphreys K, Minshew N, Leonard GL, Behrmann M (2007) A fine-grained analysis of facial expression processing in highfunctioning adults with autism. Neuropsychologia 45:685-695

24. Grüter T, Grüter M (2007) Prosopagnosia in biographies and autobiographies. Perception 36:299-301

25. von Kriegstein K, Dogan O, Grüter M, Giraud AL, Kell CA et al (2008) Simulation of talking faces in the human brain improves auditory speech recognition. Proc Natl Acad Sci USA 105:6747-6752

26. Grüter T, Grüter M, Carbon CC (2010) Congenital prosopagnosia: diagnosis and mental imagery: commentary on "Tree JJ, and Wilkie J. Face and object imagery in congenital prosopagnosia: a case series." Cortex

27. Ekman P, Friesen W (1976) Pictures of facial affects. Consulting Psychologist Press, Palo Alto

28. Cuthbert BN, Bradley MM, Lang PJ (1996) Probing picture perception: activation and emotion. Psychophysiology 33:103-111

29. Lundqvist D, Litton JE (1998) The averaged Karolinska directed emotional faces-AKDEF: psychology section. Karolinska Institutet, Stockholm

30. Kesler-West ML, Andersen AH, Smith CD, Avison MJ, Davis CE et al (2001) Neural substrates of facial emotion processing using fMRI. Brain Res Cogn Brain Res 11:213-226

31. Nakamura K, Kawashima R, Sato N, Nakamura A, Sugiura M et al (2000) Functional delineation of the human occipito-temporal areas related to face and scene processing. A PET study. Brain 123(Pt 9):1903-1912

32. Lobmaier JS, Klaver P, Loenneker T, Martin E, Mast FW (2008) Featural and configural face processing strategies: evidence from a functional magnetic resonance imaging study. Neuroreport 19:287-291

33. Good CD, Johnsrude IS, Ashburner J, Henson RN, Friston KJ et al (2001) A voxel-based morphometric study of ageing in 465 normal adult human brains. Neuroimage 14:21-36

34. Specht K, Minnerop M, Müller-Hübenthal J, Klockgether $T$ (2005) Voxel-based analysis of multiple-system atrophy of cerebellar type: complementary results by combining voxel-based morphometry and voxel-based relaxometry. Neuroimage 25:287-293

35. Friston KJ, Glaser DE, Henson RNA, Kiebel S, Phillips $\mathrm{C}$ et al (2002) Classical and Bayesian inference in neuroimaging: applications. Neuroimage 16:484-512 
36. Harmer CJ, Thilo KV, Rothwell JC, Goodwin GM (2001) Transcranial magnetic stimulation of medial-frontal cortex impairs the processing of angry facial expressions. Nat Neurosci 4:17-18

37. Grüter T, Grüter M, Bell V, Carbon CC (2009) Visual mental imagery in congenital prosopagnosia. Neurosci Lett 453:135-140

38. Behrmann M, Avidan G, Marotta JJ, Kimchi R (2005) Detailed exploration of face-related processing in congenital prosopagnosia: 1. Behavioral findings. J Cogn Neurosci 17:1130-1149

39. Yovel G, Duchaine B (2006) Specialized face perception mechanisms extract both part and spacing information: evidence from developmental prosopagnosia. J Cogn Neurosci 18:580-593

40. Stollhoff R, Jost J, Elze T, Kennerknecht I (2010) The early time course of compensatory face processing in congenital prosopagnosia. PLoS One 5(7):e11482

41. Duchaine B, Germine L, Nakayama K (2007) Family resemblance: ten family members with prosopagnosia and within-class object agnosia. Cogn Neuropsychol 24:419-430

42. Humphreys K, Minshew N, Leonard GL, Behrmann M (2007) A fine-grained analysis of facial expression processing in highfunctioning adults with autism. Neuropsychologia 45:685-695

43. Aguirre GK, Zarahn E, D'Esposito M (1998) An area within human ventral cortex sensitive to "building" stimuli: evidence and implications. Neuron 21:373-383

44. Levy I, Hasson U, Harel M, Malach R (2004) Functional analysis of the periphery effect in human building related areas. Hum Brain Mapp 22:15-26

45. Levy I, Hasson U, Avidan G, Hendler T, Malach R (2001) Center-periphery organization of human object areas. Nat Neurosci 4:533-539

46. Beauchamp MS, Lee KE, Haxby JV, Martin A (2003) FMRI responses to video and point-light displays of moving humans and manipulable objects. J Cogn Neurosci 15:991-1001

47. Gorno-Tempini ML, Price CJ (2001) Identification of famous faces and buildings: a functional neuroimaging study of semantically unique items. Brain 124:2087-2097

48. Mori S, Kaufmann WE, Davatzikos C, Stieltjes B, Amodei L et al (2002) Imaging cortical association tracts in the human brain using diffusion-tensor-based axonal tracking. Magn Reson Med 47:215-223

49. Schmahmann JD, Pandya DN (2007) The complex history of the fronto-occipital fasciculus. J Hist Neurosci 16:362-377

50. Rossion B, Caldara R, Seghier M, Schuller AM, Lazeyras F et al (2003) A network of occipito-temporal face-sensitive areas besides the right middle fusiform gyrus is necessary for normal face processing. Brain 126:2381-2395

51. Grill-Spector K, Kushnir T, Hendler T, Edelman S, Itzchak Y et al (1998) A sequence of object-processing stages revealed by fMRI in the human occipital lobe. Hum Brain Mapp 6:316-328

52. Kanwisher N, McDermott J, Chun MM (1997) The fusiform face area: a module in human extrastriate cortex specialized for face perception. J Neurosci 17:4302-4311
53. Ishai A, Schmidt CF, Boesiger P (2005) Face perception is mediated by a distributed cortical network. Brain Res Bull 67:87-93

54. Van den Stock J, van de Riet WA, Righart R, de Gelder B (2008) Neural correlates of perceiving emotional faces and bodies in developmental prosopagnosia: an event-related fMRI-study. PLoS One 3:e3195

55. Minnebusch DA, Suchan B, Koster O, Daum I (2009) A bilateral occipitotemporal network mediates face perception. Behav Brain Res 198:179-185

56. Ranganath C, Cohen MX, Dam C, D’Esposito M (2004) Inferior temporal, prefrontal, and hippocampal contributions to visual working memory maintenance and associative memory retrieval. J Neurosci 24:3917-3925

57. Druzgal TJ, D'Esposito M (2001) A neural network reflecting decisions about human faces. Neuron 32:947-955

58. Gazzaley A, Rissman J, D'Esposito M (2004) Functional connectivity during working memory maintenance. Cogn Affect Behav Neurosci 4:580-599

59. Ranganath C, Cohen MX, Brozinsky CJ (2005) Working memory maintenance contributes to long-term memory formation: neural and behavioral evidence. J Cogn Neurosci 17:994-1010

60. Kim H Dissociating the roles of the default-mode, dorsal, and ventral networks in episodic memory retrieval. Neuroimage 50: $1648-1657$

61. Blumenfeld RS, Parks CM, Yonelinas AP, Ranganath C (2011) Putting the pieces together: the role of dorsolateral prefrontal cortex in relational memory encoding. J Cogn Neurosci 23(1):257-265

62. Tree JJ, Wilkie J (2010) Face and object imagery in congenital prosopagnosia: a case series. Cortex 46(9):1189-1198

63. Bressler SL, Tang W, Sylvester CM, Shulman GL, Corbetta M (2008) Top-down control of human visual cortex by frontal and parietal cortex in anticipatory visual spatial attention. J Neurosci 28:10056-10061

64. Postle BR, Berger JS, Taich AM, D'Esposito M (2000) Activity in human frontal cortex associated with spatial working memory and saccadic behavior. J Cogn Neurosci 12(Supp12):2-14

65. Vuilleumier P, Pourtois G (2007) Distributed and interactive brain mechanisms during emotion face perception: evidence from functional neuroimaging. Neuropsychologia 45:174-194

66. Harmer CJ, Thilo KV, Rothwell JC, Goodwin GM (2001) Transcranial magnetic stimulation of medial-frontal cortex impairs the processing of angry facial expressions. Nat Neurosci 4:17-18

67. Keightley ML, Chiew KS, Anderson JA, Grady CL (2010) Neural correlates of recognition memory for emotional faces and scenes. Soc Cogn Affect Neurosci. doi:10.1093/scan/nsq003 